ARTICLE

\title{
Wall teichoic acid structure governs horizontal gene transfer between major bacterial pathogens
}

\author{
Volker Winstel ${ }^{1,2}$, Chunguang Liang ${ }^{3}$, Patricia Sanchez-Carballo ${ }^{4}$, Matthias Steglich ${ }^{5}$, Marta Munar ${ }^{3, \dagger}$, \\ Barbara M. Bröker ${ }^{6}$, Jose R. Penadés ${ }^{7}$, Ulrich Nübel ${ }^{5}$, Otto Holst ${ }^{4}$, Thomas Dandekar ${ }^{3}$, Andreas Peschel ${ }^{1,2}$ \\ \& Guoqing $X_{i a}{ }^{1,2}$
}

Mobile genetic elements (MGEs) encoding virulence and resistance genes are widespread in bacterial pathogens, but it has remained unclear how they occasionally jump to new host species. Staphylococcus aureus clones exchange MGEs such as $S$. aureus pathogenicity islands (SaPIs) with high frequency via helper phages. Here we report that the S. aureus ST395 lineage is refractory to horizontal gene transfer (HGT) with typical S. aureus but exchanges SaPls with other species and genera including Staphylococcus epidermidis and Listeria monocytogenes. ST395 produces an unusual wall teichoic acid (WTA) resembling that of its HGT partner species. Notably, distantly related bacterial species and genera undergo efficient HGT with typical S. aureus upon ectopic expression of S. aureus WTA. Combined with genomic analyses, these results indicate that a 'glycocode' of WTA structures and WTA-binding helper phages permits HGT even across long phylogenetic distances thereby shaping the evolution of Gram-positive pathogens.

\footnotetext{
${ }^{1}$ Cellular and Molecular Microbiology Division, Interfaculty Institute of Microbiology and Infection Medicine, University of Tübingen, Elfriede-Aulhorn-Straße 6, 72076 Tübingen, Germany. ${ }^{2}$ German Center for Infection Research (DZIF), partner site Tübingen, 72076 Tübingen, Germany. ${ }^{3}$ Bioinformatik, Biozentrum, University of Würzburg, Am Hubland, 97074 Würzburg, Germany. ${ }^{4}$ Division of Structural Biochemistry, Research Center Borstel, Leibniz-Center for Medicine and Biosciences, Parkallee 4a/c, 23845 Borstel, Germany. ${ }^{5}$ Robert Koch Institute 38855 Wernigerode, Germany. ${ }^{6}$ Institute of Immunology and Transfusion Medicine, University Medicine Greifswald, F.-Sauerbruchstraße, 17475 Greifswald, Germany. ${ }^{7}$ Instituto de Biomedicina de Valencia (IBV-CSIC), 46010 Valencia, Spain. †Present address: Université Libre de Bruxelles, Genomics and Structural Bioinformatics, Campus du Solbosch, Batiment U, Porte D, Niveau 3, CP165/61, Avenue F.D. Roosevelt 50, 1050 Bruxelles, Belgium. Correspondence and requests for materials should be addressed to A.P. (email: andreas.peschel@uni-tuebingen.de).
} 
$\mathrm{M}$ ajor parts of bacterial genomes consist of genetic material originating from other organisms. Many of these elements can be mobilized and exchanged by horizontal gene transfer (HGT) thereby shaping bacterial genome plasticity and permitting rapid adaptation to changing environmental challenges ${ }^{1}$. HGT of mobile genetic elements (MGEs) usually occurs at high frequency only among closely related bacterial clones because the transfer mechanisms, phagemediated transduction or plasmid conjugation, rely on specific recognition of cognate recipient strains ${ }^{1,2}$. However, HGT also occurs between members of different species or even genera albeit with lower frequency. Such rare events are responsible for the import of new genes into the species' genetic pool along with the emergence of new phenotypic properties; they are particularly important for evolution of new bacterial pathogen lineages with new virulence and antibiotic resistance traits.

The major human pathogen Staphylococcus aureus represents a paradigm for studying the roles of 'short-distance' HGT between strains of the same species and 'long-distance' HGT with other species or genera. MGEs and non-mobile genomic islands constitute ca. $22 \%$ of the $S$. aureus genomes and govern the virulence and colonization capacities, host-specificity and antibiotic resistance of the various clonal complexes ${ }^{3,4}$. Methicillinresistant $S$. aureus carrying staphylococcal cassette chromosomes with $m e c A$ genes represent the most frequent cause of severe community- or healthcare-associated infections in many developing and developed countries ${ }^{5,6}$. While conjugation and uptake of naked DNA by natural transformation seem to occur

a

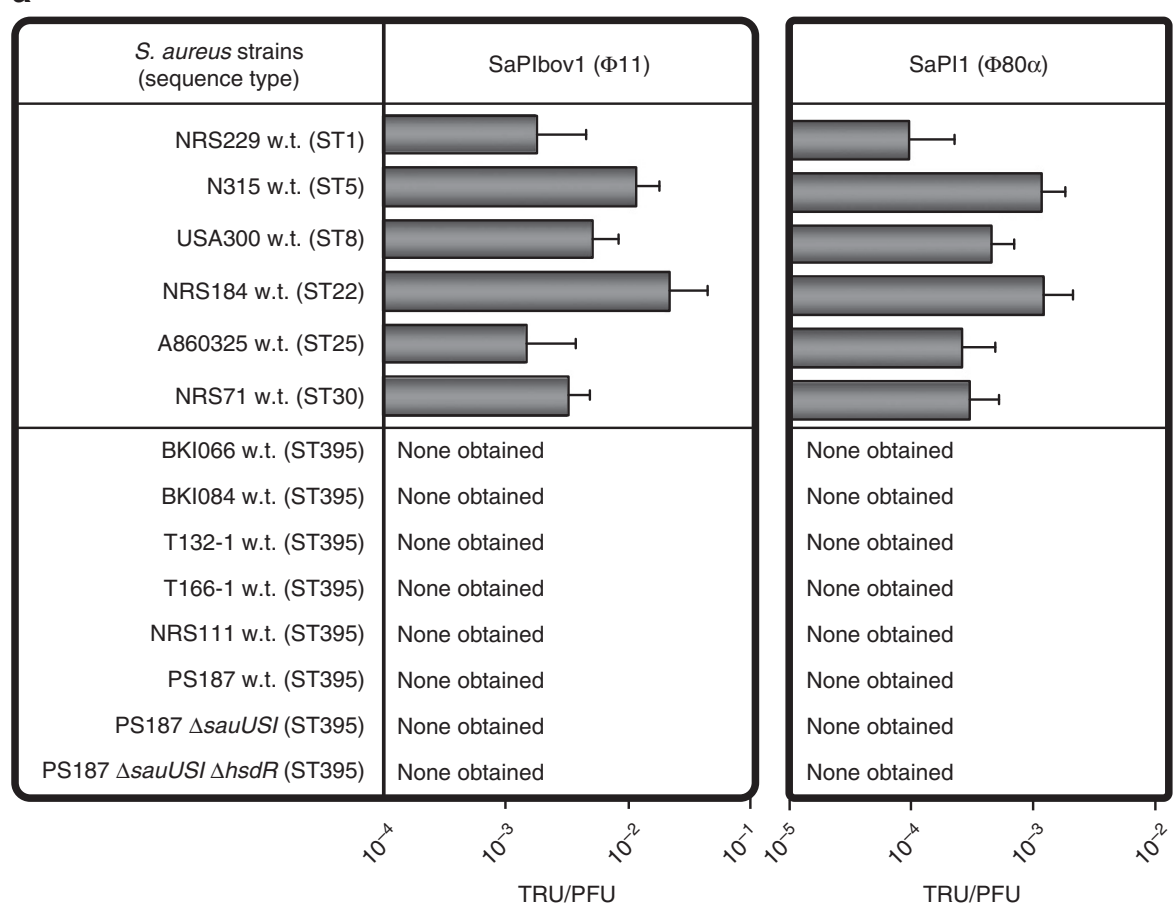

b

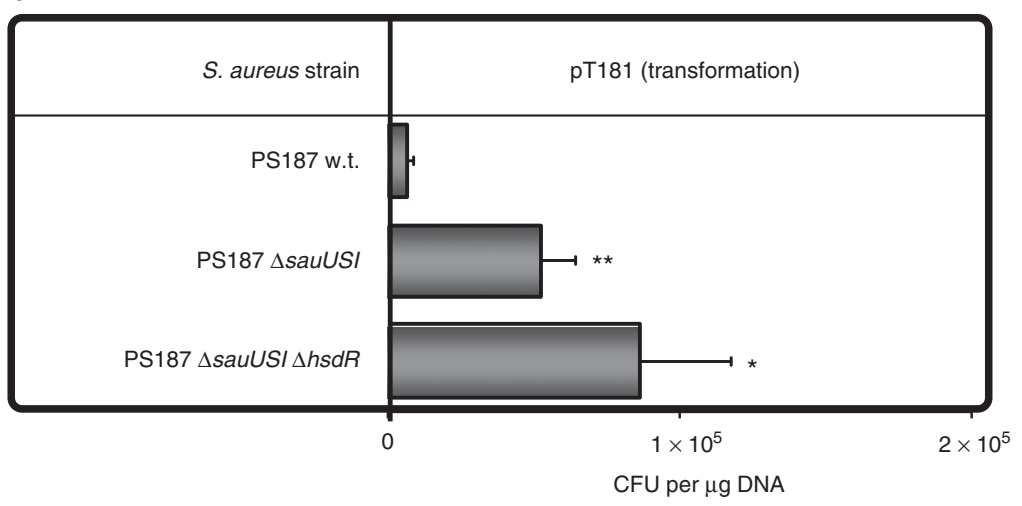

Figure 1 | ST395 is resistant to $\mathbf{\Phi 1 1}$ and $\mathbf{9 8 0} \alpha$-mediated HGT of SaPIs. (a) Various S. aureus sequence types and ST395 mutants lacking restriction modifications systems SauUSI or SauUSI plus HsdR were analysed for capacities to acquire SaPIbov1 or SaPI1 via helper phages $\Phi 11$ or $\Phi 80 \alpha$, respectively. SaPI donor strains were JP1794 (SaPIbov1) and JP3602 (SaPI1). Values represent the ratio of transduction units (TRU; transductants per ml phage lysate) to plaque-forming units (PFU; plaques per $\mathrm{ml}$ phage lysate on S. aureus RN4220 w.t.) given as means $(n=3) \pm$ s.d. No TRU were observed in controls lacking phages or SaPI particles. (b) S. aureus PS187 w.t. and mutants lacking restriction modification systems were analysed for capacities to acquire the tetracycline resistance plasmid pT181 via electroporation. Values represent the CFU per microgram DNA and given as means ( $n=3$ ) \pm s.d. CFU, colony forming units. No CFU were observed in controls lacking donor DNA. $\Delta h s d R$, no type I restriction modification system. $\Delta$ sauUSI, no type IV restriction modification system, lacks SAOUHSC_02790 homologue of NCTC8325. Statistically significant differences compared with wild type (w.t.) calculated by the unpaired two-tailed Student's $t$-test are indicated: NS, not significant, $P>0.05 ;{ }^{\star} P<0.01$ to $<0.05 ;{ }^{\star \star} P<0.001$ to $0.01 ;{ }^{\star \star \star} P<0.001$. 
rarely $^{4,7}$, staphylococcal HGT of MGEs is generally believed to depend largely on transducing helper phages ${ }^{4}$. Certain temperate phages of serogroup B such as $\Phi 11$ or $\Phi 80 \alpha$ have been shown to be capable of transducing DNA between $S$. aureus clones and to employ the $N$-acetyl-D-glucosamine (GlcNAc) residues on wall teichoic acid (WTA), a surface-exposed glycopolymer, as receptor ${ }^{4,8}$. WTA is produced by most Gram-positive bacteria and usually has species- or strain-specific structure ${ }^{9}$. S. aureus produces a WTA polymer composed of ca. 40 ribitol-phosphate (RboP) repeating units modified with $\alpha$ - and/or $\beta$-linked GlcNAc and D-alanine ${ }^{9,10}$ while the various coagulase-negative staphylococcal species (CoNS) produce WTA with glycerophosphate (GroP) or hexose-containing, complex repeating units modified with different types of sugars ${ }^{11}$.

S. aureus pathogenicity islands (SaPIs) are exchanged among $S$. aureus lineages with high frequency by SaPI particles consisting of SaPI genomes and structural proteins from helper phages ${ }^{12,13}$. While such 'short-distance' HGT events occur with high frequency, antibiotic resistance-mediating MGEs have been acquired only occasionally from other bacterial species. Of note, $\beta$-lactam antibiotic resistance genes from CoNS have frequently been imported into $S$. aureus ${ }^{14}$ whereas enterococcal vancomycin resistance genes have emerged in staphylococci only in a few exceptional cases ${ }^{15}$ suggesting that there are mechanisms involved that favour or disfavour specific 'long-distance' HGT events. Restriction modification systems ${ }^{16-18}$ and rarely occurring clustered, regularly interspaced, short palindromic repeat (CRISPR) sequences ${ }^{19}$ have been shown to interfere with HGT efficiency in staphylococci but the major determinants permitting HGT with other bacterial species and genera have remained unknown.

We demonstrate here that the variable structure of glycosylated WTA constitutes a 'glycocode' that is sensed by transducing phages thereby defining the routes of HGT. Similar WTA structures enable DNA exchange via helper bacteriophages even across the boundaries of species or genera, whereas $S$. aureus clones producing altered WTA become separated from the species' genetic pool and may initiate new routes of HGT with other bacterial species and genera that share related WTA. Thus, related WTA structures are sufficient to initiate HGT even across long phylogenic distances.

\section{Results}

ST395 cannot undergo HGT with other $S$. aureus lineages. The various $S$. aureus clonal complexes differ largely in their epidemic potential and number of MGEs ${ }^{4}$. We compared several S. aureus lineages for capability to acquire SaPI1 or SaPIbov1 originating from sequence types ST8 and ST151, respectively ${ }^{13}$. Derivatives of these SaPIs with antibiotic resistance gene markers ${ }^{20}$ were transferred from $S$. aureus ST8 to a variety of potential recipient strains using helper phages $\Phi 11$ (for SaPIbov1) or $\Phi 80 \alpha$ (for SaPI1). The majority of the sequence types acquired SaPIs albeit with varying efficiency, probably as a consequence of different restriction modification systems ${ }^{16-18}$ (Fig. 1a). In contrast, several independent clones of the ST395 lineage from various parts of the world including isolates from the lung or blood stream infections and nasal swabs (Supplementary Table S1) ${ }^{21-23}$ were completely resistant to HGT of SaPIs (Fig. 1a). Restriction modification systems were obviously not responsible for HGT resistance of ST395 because consecutive inactivation of the genes for type I $(\Delta h s d R)$ and type IV ( $\Delta$ sauUSI) restriction systems in the ST395 strain PS187 did not enable transfer of SaPIs (Fig. 1a), whereas it considerably increased the rates of plasmid electroporation (Fig. 1b).

Ф187 mediates HGT between ST395 and other bacterial species. While strain PS187 was resistant to infection by $\Phi 80 \alpha$ or $\Phi 11$ (Supplementary Fig. S1a), it has previously been shown to be susceptible to phage $\Phi 187$ (refs 21,24). Interestingly, most ST395

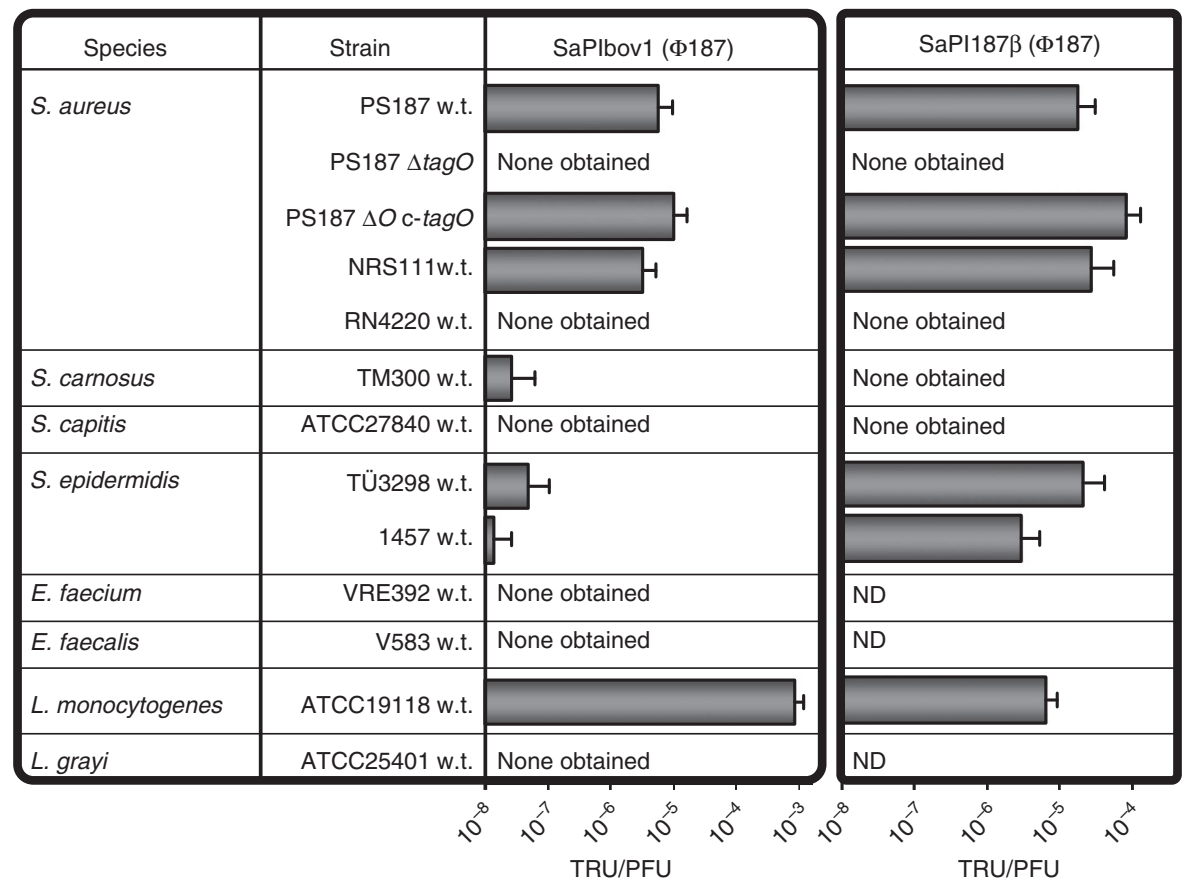

Figure 2 | 1818 mediates HGT of SaPIs among ST395 and to other species and genera. S. aureus ST395 strains with or without WTA or other Gram-positive bacteria were analysed for capacities to acquire SaPIbov1 or SaPl187 $\beta$ via helper phage $\Phi 187$. SaPI donor strains were VW1 (SaPIbov1) and VW7 (SaPI187 $\beta$ ). Values represent the ratio of transduction units (TRU; transductants per $\mathrm{ml}$ phage lysate) to plaque-forming units (PFU; plaques per $\mathrm{ml}$ phage lysate on S. aureus PS187 w.t.) given as means $(n=3) \pm$ s.d. No TRU were observed in controls lacking phages or SaPI particles. $\Delta$ tagO, no WTA. c-tagO, $\Delta$ tagO complemented with tagO. ND, not determined due to marker resistance or spontaneous occurring clones. 
but none of the other $S$. aureus sequence types could be infected by $\Phi 187$ (Supplementary Figs S1a and S2a). When $\Phi 187$ was analysed for its capacity to transfer MGEs, it was found to facilitate indeed the exchange of SaPI187 $\beta$ (found in the PS187 genome, see below) and SaPIbov1 between different ST395 isolates but not to other $S$. aureus sequence types (Fig. 2). Surprisingly, it also mediated HGT of SaPIbov1 and SaPI187 $\beta$ from ST395 to the CoNS species Staphylococcus epidermidis and Staphylococcus carnosus and even to Listeria monocytogenes serotype 4e (Fig. 2). Thus, ST395 can participate in HGT with other species and genera while it is separated from phage-dependent HGT with typical $S$. aureus.

ST395 has a unique WTA gene cluster and WTA structure. PS187 was sequenced to obtain a prototype ST395 genome. The draft sequence was assembled into 16 large contigs plus two plasmids encompassing 2,529 open reading frames along with 43 and 10 coding sequences for transfer RNAs and ribosomal ribonucleic acid RNAs, respectively. PS187 was found to be a true
S. aureus but to branch deeply in the $S$. aureus lineage (Fig. 3a). It did not encode CRISPR determinants indicating that the unusual HGT behaviour of ST395 is probably not mediated by 'adaptive immunity' to foreign DNA ${ }^{19}$. Among several unusual MGEs (see below) PS187 contained a novel genomic element, which encompassed transposon-related sequences plus four genes with similarity to WTA-biosynthetic genes $(\operatorname{tag} V, \operatorname{tag} N, \operatorname{tag} D, \operatorname{tag} F)$ (Fig. 3b). Of note, the new element replaced the $11-\mathrm{kb}$ tarIJLFS cluster for biosynthesis of GlcNAc-modified RboP (RboPGlcNAc) WTA ${ }^{10}$, which is found in all other so far known $S$. aureus genomes. Different ST395 clones were found to be related because they exhibited similar DNA fragment patterns in pulsed field gel electrophoreses albeit with some variation in the size of certain DNA bands (Supplementary Fig. S2b). Moreover, all of the 10 available ST395 isolates were tested positive for the $\operatorname{tag} N$ gene encoded on the new genetic element indicating that the new WTA gene cluster is probably a common feature in the ST395 lineage (Supplementary Fig. S2c). WTA was isolated from PS187 and NMR-based structural elucidation demonstrated that PS187

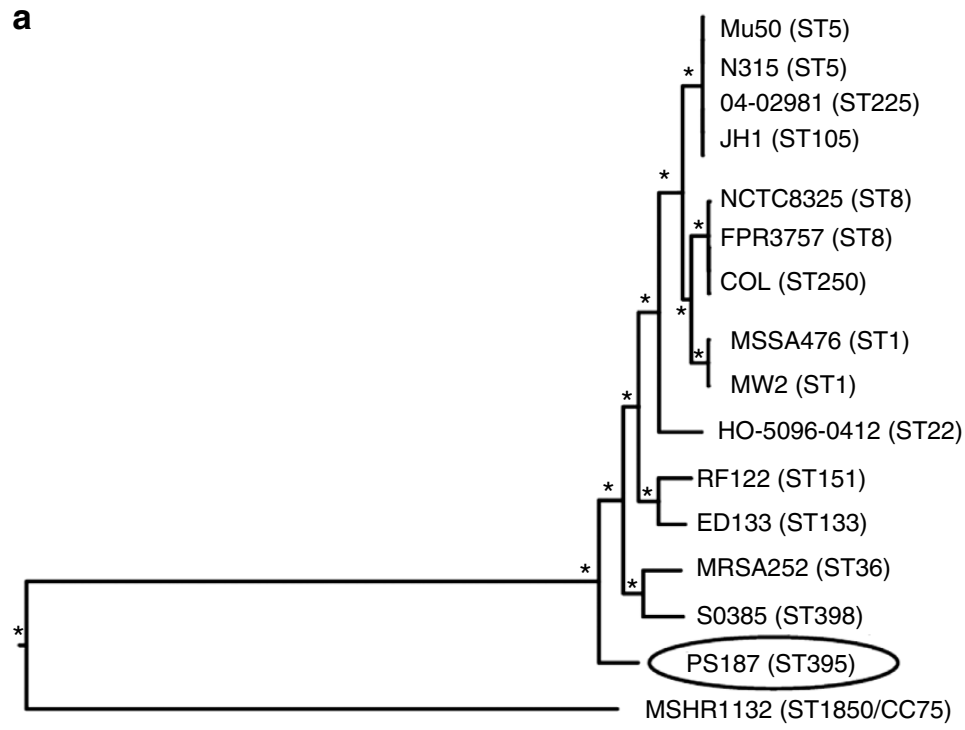

C
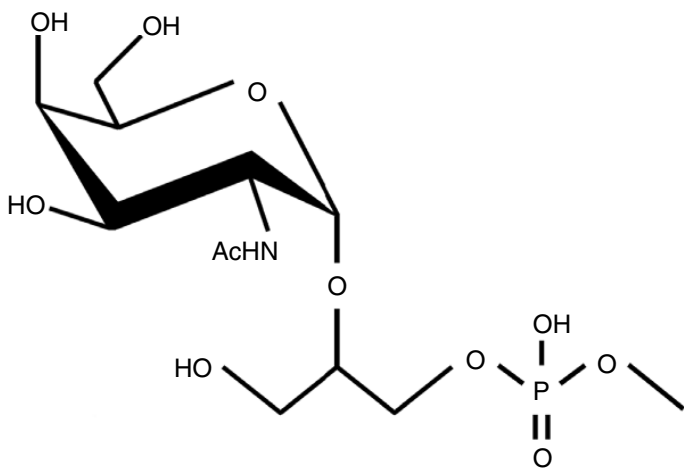

$10, \overline{000 \text { SNPS }}$

b

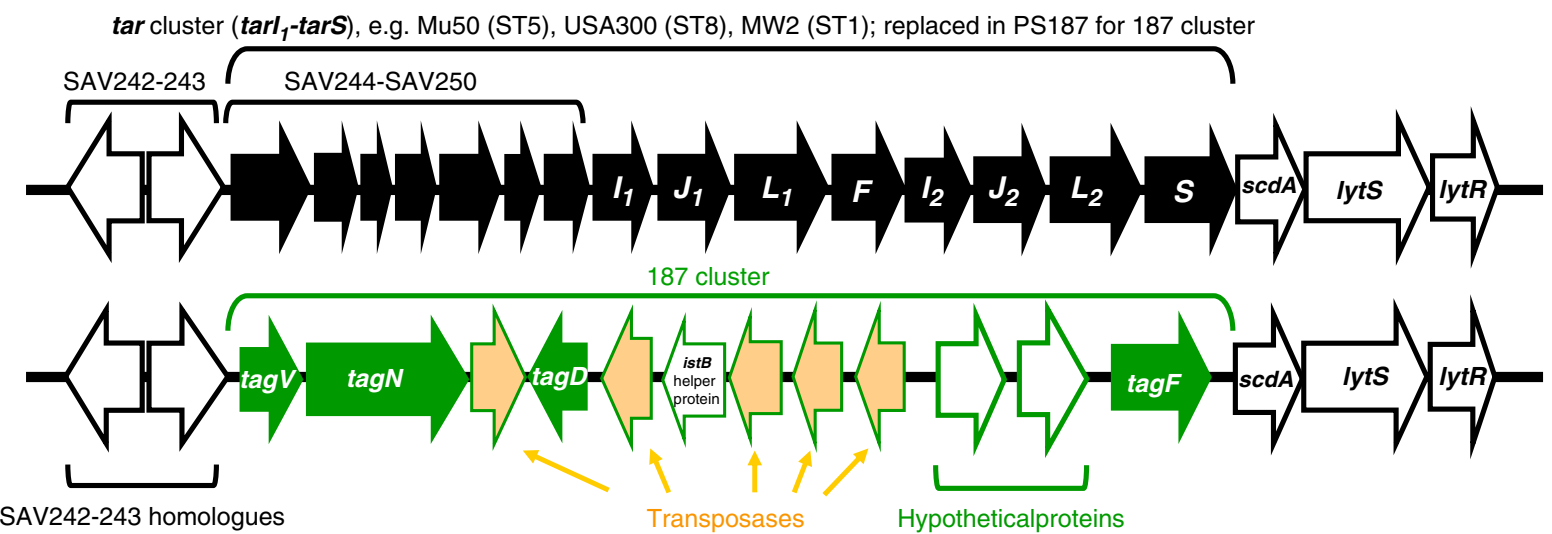

Figure 3 | ST395 branches deeply from other S. aureus lineages and bears a novel WTA type and WTA gene cluster. (a) Phylogenetic relationships of S. aureus sequence types based on DNA sequences from 1,147 orthologous genes. The genome of $S$. epidermidis RP62A was used for rooting the tree. Asterisks indicate 100\% branch support in both, the maximum-likelihood tree and the Bayesian maximum clade credibility tree. (b) ST395 bears a novel WTA-biosynthetic gene cluster. Genetic organization of the RboP-GlcNAc WTA-biosynthetic tar cluster found in all S. aureus genomes (upper cluster) that is replaced by a new gene cluster containing putative WTA-biosynthetic genes (green) in ST395 strain PS187 (lower cluster). Gene locus numbers are indicated. Protein sequence alignments of the characteristic ST395 WTA proteins with homologues from CoNS can be found in Supplementary Fig. S6. (c) The chemical structure of the WTA repeating unit of S. aureus strain PS187 WTA (GroP- $\alpha-D-G a l p N A c$ ). 
produces a unique WTA type with an $N$-acetyl-D-galactosamine (GalNAc) modified GroP backbone (GroP-GalNAc) (Fig. 3c; Supplementary Fig. S3a and Supplementary Table S2), which was in agreement with an earlier analysis of PS187-related strains ${ }^{11}$.

Some unusual MGEs from ST395 may be originating from CoNS. Because of its inability to exchange DNA with typical S. aureus, we assumed that ST395 may be genetically isolated. ST395 clones have been occasionally described as human commensals and invasive pathogens ${ }^{22,23}$ and a recent study has found that ST395 accounts for 5 and $2 \%$ of S. aureus nasal and blood culture isolates, respectively, in Northeastern Germany ${ }^{23}$. However, the actual numbers may be higher because ST395 isolates are usually methicillin susceptible and such clones are hardly collected and typed. Interestingly, early reports from the 1960s have pointed to a canine reservoir of PS187-related S. aureus $^{25}$. In accord with this notion, we found the sequences of some of the host range-determining proteins of PS187 (IsdB and $\mathrm{VwBP})^{26}$ to differ from typical human strains whereas the presence of the strictly human-specific chp, scn and sak genes ${ }^{26}$ suggests that PS187 is at least in part adapted to the human host.

Most MGEs of PS187 were distinct in synteny and composition from those found in other $S$. aureus genomes and some were even more related to MGEs found in CoNS. The genomic islands vSA $\alpha$ and vSA $\beta$ found in all previously sequenced $S$. aureus genomes were also present in PS187 but lacked typical enterotoxin or lantibiotic gene clusters, respectively (Fig. $4 \mathrm{a}$ and Supplementary Fig. S4). Two novel PS187 SaPIs named SaPI187 $\alpha$ and SaPI187 $\beta$

a
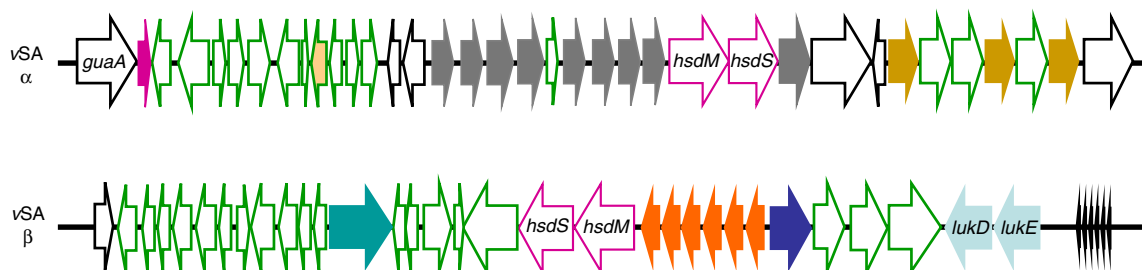

b

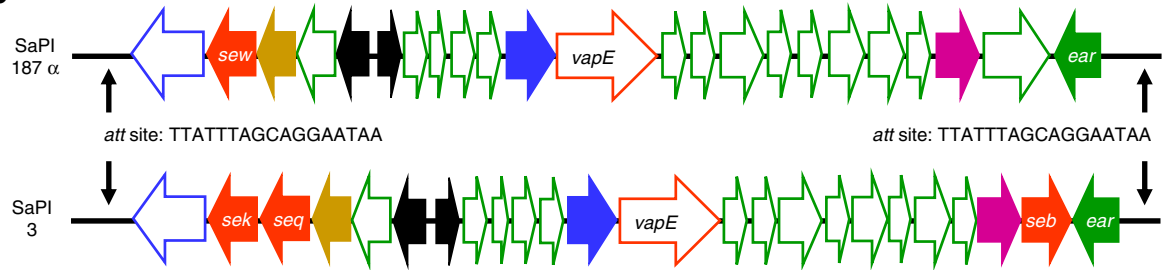

C

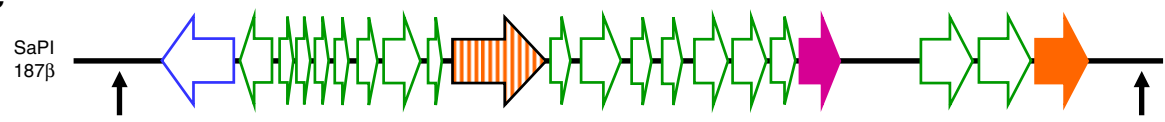

att site: GTTTTACATCATTCCTGGCAT att site: GTTTTACATCATTCCTGGCAT

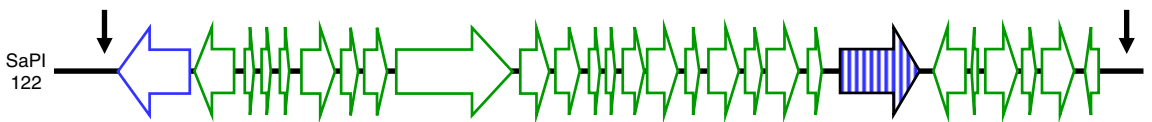

d
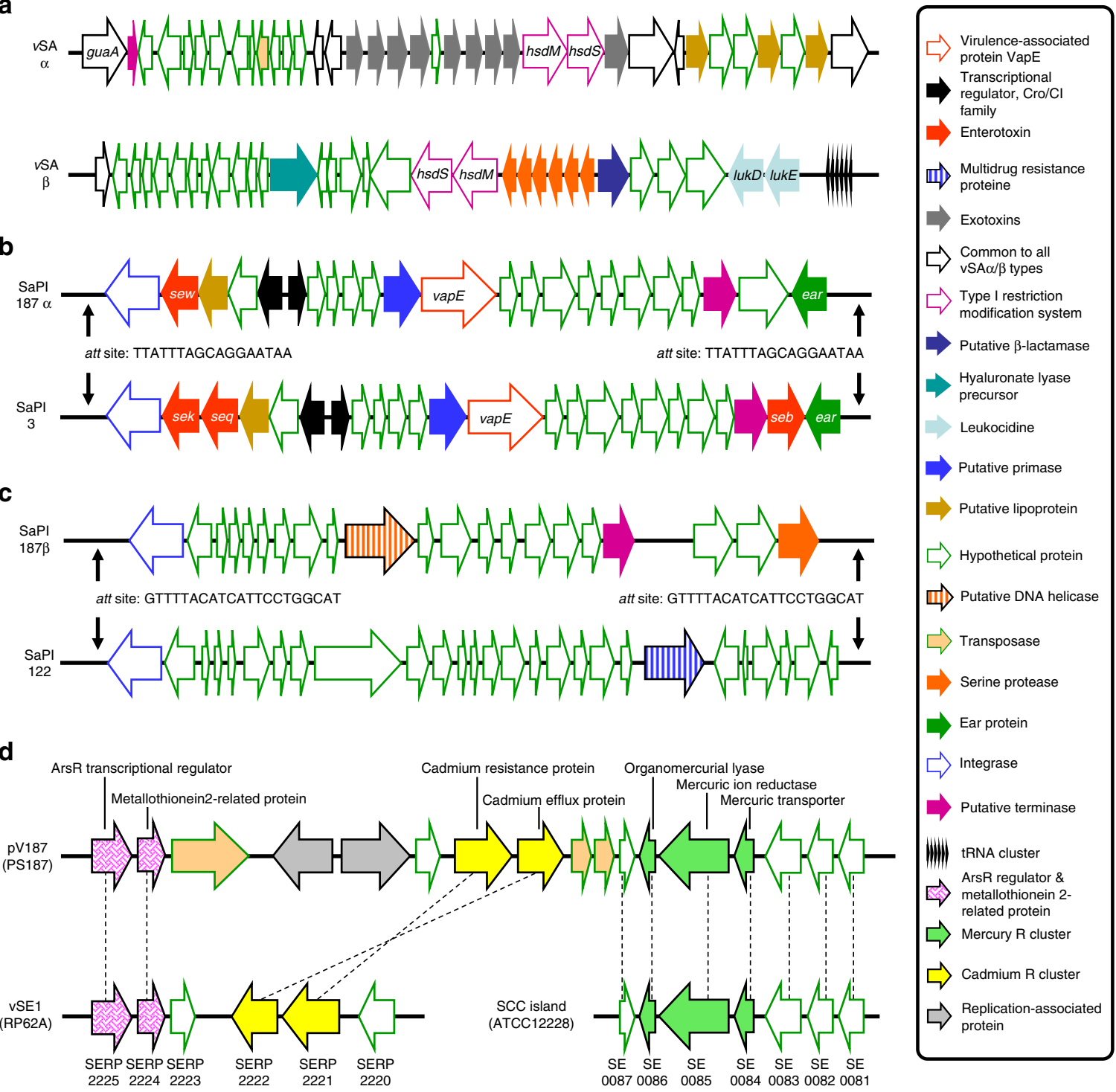

Figure 4 | Characteristic MGEs of S. aureus PS187. (a) Genetic organization of the two genomic islands found in PS187. The organization of PS187 vSA $\alpha$ is similar to that of other $S$. aureus genomes. The vSA $\beta$ of PS187 lacks typical enterotoxin or lantibiotic clusters found in all other vSA $\beta$ types sequenced so far. (b) Comparison of SaPI187 $\alpha$ from PS187 and SaPI3 from typical S. aureus genomes. SaPI187 $\alpha$ shares the att site with SaPI1, SaPI3 and SaPI5 and was found to be similarly organized as SaPI3 from S. aureus Col. Note the presence of a novel enterotoxin in SaPI187 $\alpha$ designated as enterotoxin SeW. (c) Comparison of SaPI187 $\beta$ from PS187 and SaPI122 from S. aureus RF122. Although SaPI187 $\beta$ shares the att site with SaPI122 the SaPI122-characteristic gene encoding a multidrug resistance protein is absent in SaPI187 $\beta$. Instead SaPI187 $\beta$ encodes a new serine protease. (d) Comparative map of mercury and cadmium resistance operons on a large plasmid from PS187 and corresponding genes from the S. epidermidis ATCC12228 SCC composite island (right) and the S. epidermidis RP62A integrated plasmid vSE1 (left). Dotted lines indicate sequence identities above >92\% (DNA level) and 93\% (protein level). Gene locus numbers are listed and ORFs are coloured according to functional categories as indicated. 
shared attachment (att) sites and some similarity with $\mathrm{SaPI} 3$ and SaPI122, respectively ${ }^{13}$, but differed with regard to enterotoxin, multidrug resistance transporter and serine protease genes (Fig. 4b,c and Supplementary Fig. S4). PS187 was found to encode two cryptic prophages named $\Phi$ PS187a and ФPS187b (Supplementary Fig. S4), which were similar to previously described S. aureus phages $\Phi 77$ (ref. 8) and $\Phi 187$ (refs 21,24) and were integrated in the genes for sphingomyelinase $(h l b)$ and the giant surface protein Ebh, respectively. However, both prophages were defective as no infective phages could be obtained from PS187 upon treatment with the prophage-inducing agent mitomycin C (Supplementary Fig. S5). $\Phi 77$ was found to have entirely different host binding specificities from $\Phi 187$ with efficient adsorption to $S$. aureus with RboP-GlcNAc WTA and inefficient binding to bacteria with other WTA types such as ST395 (Supplementary Fig. S1b) suggesting that the defective $\Phi 77$-related prophage of PS187 may have originated from an ancient HGT event before the new WTA-biosynthetic genes had been acquired.

Notably, some of the PS187 MGEs shared higher similarity with genes from CoNS than from other $S$. aureus. This was found for mercury and cadmium resistance operons on a large plasmid, which were most similar to corresponding genes from an S. epidermidis SCC island ${ }^{27}$ and the chromosomally integrated S. epidermidis plasmid $\mathrm{vSe}^{28}$ (Fig. $4 \mathrm{~d}$ ), respectively, and for the new WTA-biosynthetic genes, whose products exhibited highest similarity with proteins from Staphylococcus pseudintermedius (TagV), S. carnosus (TagN), Staphylococcus lugdunensis (TagD), and Staphylococcus simiae (TagF) (Supplementary Fig. S6). Thus, the genome sequence confirms that ST395 clones are separated from frequent HGT with typical $S$. aureus but have access to MGEs from CoNS.

SaPI particles adopt receptor requirements of helper phages. The unusual WTA structure of PS187 resembled that of the CoNS strains accepting SaPI DNA from ST395 via $\Phi 187$ (Table 1) suggesting that similar WTA structure may be a crucial determinant for the initiation of phage-dependent gene transfer even between distantly related bacteria. However, while S. aureus $\Phi 80 \alpha$ or $\Phi 11$ phage particles are known to require a defined WTA structure for adsorption to host bacteria $^{8}$, the receptor requirements of SaPI particles, which have a much broader host range than helper phages ${ }^{29}$, has remained unknown. Using defined mutants of the ST8 strains RN4220 (refs 8,10) and Newman with altered teichoic acids, the susceptibilities to phage $\Phi 80 \alpha$ or $\Phi 11$ particle binding and infection were compared with their capacities to acquire SaPI DNA from SaPI particles derived from the same helper phages. Infection by both, phage particles and SaPI particles, was dependent on the presence of WTA and WTA glycosylation (Fig. 5a; Supplementary Fig. S7) whereas lipoteichoic acid was dispensable (Fig. 5a) indicating that SaPI particles adopt the receptor requirements of the corresponding helper phage. In accord with this finding, inactivation of tagO encoding the first enzyme of the WTA-biosynthetic pathway rendered strain PS187 resistant to $\Phi 187$ infection because of impaired adsorption (Supplementary Fig. 1a,b) and to $\Phi 187$ dependent SaPI transfer (Fig. 2) indicating that $\Phi 187$ and $\Phi 187$ derived SaPI particles require GroP WTA for binding to host bacteria.

WTA structure governs the capacity to undergo HGT. In order to elucidate if it is indeed the similarity of WTA structures that determines if two bacterial strains can exchange DNA via helper phages, even if they are not closely related, we cloned the minimal set of genes (tarFIJLS) from S. aureus RN4220 (ST8) required for biosynthesis of RboP-GlcNAc WTA ${ }^{10}$ and introduced the resulting plasmid into bacteria that were not susceptible to HGT with typical RboP-WTA expressing $S$. aureus. The plasmidtransformed strain PS187-H was indeed found to produce two types of WTA as NMR analysis revealed the presence of both, RboP-GlcNAc WTA and GroP-GalNAc WTA (Supplementary Fig. S3b and Supplementary Table S3) indicating that two different WTA-biosynthetic machineries can be simultaneously functional in bacterial cells. Growth or morphology of these hybrid strains did not show obvious alterations (Supplementary Fig. S8a,b). Indeed, upon expression of RboP-GlcNAc WTA ST395 strains PS187 and NRS111 became susceptible to $\Phi 80 \alpha$ or Ф11-dependent HGT of SaPI1 and SaPIbov1, respectively (Fig. 5b) or of the tetracycline resistance plasmid pT181 (Supplementary Fig. S9) from typical S. aureus. Moreover, distantly related bacteria such as S. epidermidis, S. carnosus, S. capitis, and even L. monocytogenes serotype $4 \mathrm{e}$, which are usually resistant to $\Phi 80 \alpha$ or $\Phi 11$-dependent HGT, acquired the capacity to take up SaPIs from typical $S$. aureus upon expression of tarFIJLS at similarly high rates as RboP-GlcNAc WTA-producing S. aureus (Fig. 5b). Of note, successful SaPI transfer correlated with increased phage adsorption upon RboP-GlcNAc WTA expression (Supplementary Fig. S1c). These results demonstrate that related WTA structures can be sufficient for allowing MGE exchange via helper phages even across long phylogenetic distances.

\begin{tabular}{|c|c|c|c|c|c|}
\hline Species/Strain & WTA type ${ }^{\star}$ & Glycosylation $^{\star}$ & Reference & $\begin{array}{c}\text { Transduction } \\
\text { from ST8 via } \\
\Phi 11 / 80 \alpha\end{array}$ & $\begin{array}{c}\text { Transduction } \\
\text { from ST395 via } \\
\text { Ф187 }\end{array}$ \\
\hline S. aureus RN4220 (ST8) & RboP & $\operatorname{GIcNAc}(\alpha), \operatorname{GIcNAc}(\beta)$ & 38 & + & - \\
\hline S. aureus PS187 (ST395) & GroP & GalNAc $(\alpha)$ & This study & - & + \\
\hline S. epidermidis $1457^{\dagger}$ & GroP & $\operatorname{GlcNAc}(\alpha) ; \mathrm{Glc}$ & 11 & - & + \\
\hline E. faecalis V $583^{\ddagger}$ & $\begin{array}{l}\text { GalNAc-RboP, WTA1 } \\
\text { Glc-GalNAc-RboP, WTA2 }\end{array}$ & $\begin{array}{l}\text { Rha }(\alpha) \text {, WTA1 Glc }(\alpha) \text {, } \\
\text { WTA2 }\end{array}$ & 31 & - & - \\
\hline $\begin{array}{l}\text { L. monocytogenes } \\
\text { ATCC19118 } \dagger \text {, Serotype } 4 \mathrm{e}\end{array}$ & GlcNAc-RboP & Gal & 43 & - & + \\
\hline L. grayi ATCC25401 & RboP & GIcNAc & 43 & + & - \\
\hline
\end{tabular}


a
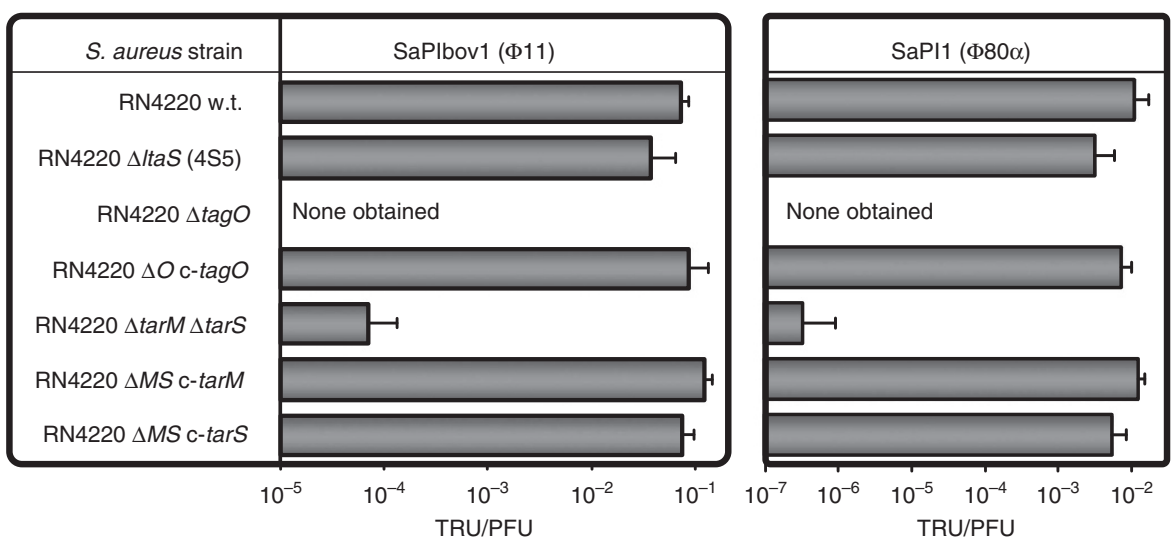

b

\begin{tabular}{|c|c|c|c|}
\hline Species & Strain & SaPIbov1 (Ф11) & SaPI1 $(\Phi 80 \alpha)$ \\
\hline \multirow[t]{4}{*}{ S. aureus } & PS187 w.t. & None obtained & None obtained \\
\hline & PS187-H & & \\
\hline & NRS111 w.t. & None obtained & None obtained \\
\hline & NRS111-H & - & 1 \\
\hline \multirow[t]{2}{*}{ S. carnosus } & TM300 w.t. & None obtained & None obtained \\
\hline & TM300-H & & \\
\hline \multirow[t]{2}{*}{ S. capitis } & ATCC27840 w.t. & None obtained & None obtained \\
\hline & ATCC27840-H & & \\
\hline \multirow[t]{2}{*}{ S. epidermidis } & 1457 w.t. & None btained & None obtained \\
\hline & $1457-\mathrm{H}$ & & \\
\hline \multirow[t]{2}{*}{ L. monocytogenes } & ATCC19118 w.t. & None obtained & None obtained \\
\hline & ATCC19118-H & & H \\
\hline \multirow[t]{2}{*}{ L. grayi } & ATCC25401 w.t. & H & None obtained \\
\hline & & TRU/PFU & $\begin{array}{l}\hat{0}^{3} \\
\text { TRU/PFU }\end{array}$ \\
\hline
\end{tabular}

Figure 5 | WTA structure determines the capacity of SaPls to traverse even long phylogenetic distances. S. aureus RN4220 (ST8) strains with, with altered or without WTA (a) or S. aureus PS187 (ST395) or other Gram-positive bacterial species expressing genes for biosynthesis of RboP-GlcNAc WTA (b) were analysed for capacities to acquire SaPIbov1 or SaPI1 via helper phages $\Phi 11$ or $\Phi 80 \alpha$, respectively. SaPI donor strains were JP1794 (SaPIbov1) and JP3602 (SaPI1). Values represent the ratio of transduction units (TRU; transductants per ml phage lysate) to plaque-forming units (PFU; plaques per $\mathrm{ml}$ phage lysate on S. aureus RN4220 w.t.) given as means $(n=3) \pm$ s.d. No TRU were observed in strains expressing WTA other than RboP-GlcNAc. $\Delta \operatorname{tag} \mathrm{O}$, no WTA; $\Delta$ taS (4S5), no lipoteichoic acid; c-tagO, $\Delta \operatorname{tagO}$ complemented with tagO; $\Delta \operatorname{tarM} \Delta \operatorname{tarS}$, no WTA glycosylation; c-tarM and c-tarS, $\Delta$ tarM $\Delta$ tarS complemented either with tarM or tarS. WTA hybrid strains expressing additional RboP-GlcNAc WTA are indicated with H.

In accord with this finding, a systematic analysis with several Gram-positive bacteria revealed a strong correlation between WTA structure and the capacity to exchange resistance and virulence genes with either typical S. aureus via $\Phi 11$ or $\Phi 80 \alpha$ (for example, Listeria grayi) or with ST395 via $\Phi 187$ (for example, S. epidermidis and other CoNS) (Table 1). The susceptibility of L. monocytogenes serotype $4 \mathrm{e}$ to $\Phi 187$-mediated HGT was reflected by efficient binding of $\Phi 187$ (Supplementary Fig. S1b). It may be due to the decoration of WTA with galactose, which may facilitate binding of $\Phi 187$-derived SaPI particles in a similar way as GalNAc. Of note, vancomycin-resistant Enterococcus faecium or Enterococcus faecalis could not undergo HGT with any $S$. aureus probably because of the very complex enterococcal WTA structures ${ }^{30,31}$.

\section{Discussion}

HGT between $S$. aureus and other bacterial species and genera contributes substantially to the evolution of new epidemic clones, but it has remained unclear if it occurs accidentally or follows certain rules. While restriction modification and CRISPR systems have been shown to limit HGT efficiency ${ }^{16-19}$, no data on the criteria that need to be fulfilled by the HGT partners to initiate phage-dependent MGE exchange have been available. Our studies with naturally occurring and engineered bacterial strains with atypical WTA demonstrate that related WTA structures of MGE donor and recipient are sufficient to permit HGT even across long phylogenetic distances. While helper phage particles are known to have quite narrow host ranges for parasitic reproduction ${ }^{29}$, we found that their receptor specificities govern the capacity to 
transmit MGEs to a broad range of recipient strains expressing cognate surface ligands. On the other hand, changes in helper phage receptor structures can enable binding of new types of helper phages and redirect the routes of HGT, which may facilitate the development of new clonal lineages and species.

The structurally diverse WTA molecules represent a species or lineage-specific signature at the surface of Gram-positive bacteria. WTA has several important roles for bacterial physiology such as the control of autolytic and peptidoglycan-biosynthetic enzymes? but all these functions could be achieved without extensive variation of its structure. Our data indicate that WTA structure constitutes a species- or lineage-defining 'glycocode' governing the bacterial access to a common genetic pool via WTA-specific helper phages. A thorough investigation of WTA structures and cognate phage receptor specificities will enable to assess how likely the transfer of new antibiotic resistance genes across the boundaries of species and genera will be in the future. Antibiotic stress is known to activate prophages thereby probably contributing to phage-mediated $\mathrm{HGT}^{32}$. In contrast, recently developed compounds blocking the biosynthesis of $\mathrm{WTA}^{33}$ may help to reduce the frequency of phage-dependent HGT for example, in chronic polymicrobial infections.

\section{Methods}

Bacterial strains and growth media. The various bacterial strains listed in Supplementary Table S1 were grown in BM broth (1\% tryptone, $0.5 \%$ yeast extract, $0.5 \% \mathrm{NaCl}, 0.1 \% \mathrm{~K}_{2} \mathrm{HPO}_{4}, 0.1 \%$ glucose) or Luria Bertani Broth supplemented with appropriate antibiotics at a concentration of $10 \mu \mathrm{g} \mathrm{ml}^{-1}$ (chloramphenicol), $3 \mu \mathrm{g} \mathrm{ml}^{-1}$ (tetracycline) or $100 \mu \mathrm{g} \mathrm{ml}^{-1}$ (ampicillin). In order to assess the growth and microscopic properties of mutant strains, phenotypic characterization of WTA hybrid strains overnight cultures were diluted to $\mathrm{OD}_{578} 0.1$ in $\mathrm{BM}$ and grown at $37^{\circ} \mathrm{C}$ on a shaker. Samples for microscopy were analysed after 3-h growth using a Leica DMRE microscope and Leica HCX $100 \times$ objective. Bacterial growth was monitored for $8 \mathrm{~h}$

Molecular genetic methods. For the construction of marker-less $\Delta$ tagO, $\Delta$ sauUSI and $\Delta$ sauUSI $\Delta h s d R$ mutants in $S$. aureus PS187, the pKOR1 shuttle vector was used according to standard procedures ${ }^{34}$. For knockout plasmid construction, primers listed in Supplementary Table S4 were used. To label SaPI187 $\beta$ with an antibiotic resistance marker, the ermB gene was integrated into SaPI187 $\beta$ using the pKOR1 system. Knock-in primers are listed in Supplementary Table S4.

For amplification of the genomic region coding for $\operatorname{tar} F, \operatorname{tarI}_{2}, \operatorname{tarI}_{2}, \operatorname{tar}_{2}$ and tarS from genomic DNA (RN4220 w.t.) primers, Tar-up and Tar-dn were used. The PCR product was cloned in the E. coli/S. aureus shuttle vector pRB $474^{35}$ at the BamHI/EcoRI restriction sites resulting in plasmid pRB474-tarFI $J_{2} L_{2} S$. The plasmid was isolated from E. coli TOP10 or E. coli DC10B and used to transform target strains resulting in hybrid WTA-producing strains $S$. aureus PS187-H and NRS111-H, S. carnosus TM300-H, S. capitis ATCC27840-H and S. epidermidis 1457-H. L. monocytogenes ATCC19118 was transformed by electroporation ${ }^{36}$ resulting in strain L. monocytogenes ATCC19118-H.

For plasmid transformation, $50 \mu \mathrm{l}$ competent cells were mixed with $500 \mathrm{ng}$ pT181 plasmid DNA, incubated at room temperature for $30 \mathrm{~min}$, transferred into a 1-mm gap electroporation cuvette and pulsed at 1,000 volts. Immediately after electroporation, $950 \mu \mathrm{l} \mathrm{BM}$ medium was added followed by 70 -min incubation at $37^{\circ} \mathrm{C}$. Cells were finally plated onto selective media and transformants were counted to calculate electroporation efficiency.

Pulsed field gel electrophoresis typing was performed according to Goerke et al. ${ }^{37}$ tagN genotyping of available ST395 isolates was performed using primers $\mathrm{N}$-up and N-dn (Supplementary Table S4).

Experiments with phages and SaPI particles. For rapid determination of phage susceptibility, the soft agar spot assay was performed according to Xia et al. ${ }^{38} \mathrm{~A}$ phage panel encompassing broad host range phage $\Phi \mathrm{K}$ (ref. 38), serogroup L phage $\Phi 187$ (ref. 39), two serogroup B phages $\Phi 11$ (ref. 39) and $\Phi 80 \alpha^{40}$ and serogroup F phage $\Phi 77$ (ref. 8) (Supplementary Table S5) was used. The various phages were propagated on S. aureus RN4220 (ФK, Ф11, Ф80 $\alpha$, Ф77) or PS187 (Ф187). Mitomycin C induction experiments confirmed that these two propagation strains released no phage particles from endogenous prophages thereby ensuring that no other than the propagated phages were present in the obtained lysates. In order to determine phage adsorption rates, the multiplicity of infection was set to 0.005 (for $\Phi 187$ ) or 0.1 (for $\Phi 80 \alpha$ and $\Phi 77$ ). Adsorption was calculated by determining the PFU of the unbound phage in the supernatant and subtracting it from the total number of input PFU. Adsorption efficiency was indicated relative to the adsorption on the parental wild-type strain (RN4220 or PS187), which was set to $100 \%$. Prophages or SaPI particles were induced with $1 \mu \mathrm{g} \mathrm{ml}^{-1}$ mitomycin C.

For phage-mediated SaPI and plasmid transfer, recipient strains were grown overnight and used for HGT experiments. Approximately $8.0 \times 10^{7}$ bacteria were mixed with $100 \mu \mathrm{l}$ of SaPI lysates produced from S. aureus strains JP1794 or JP3602 bearing the resistance marker-labelled SaPIbov1 $\left(\sim 1.0 \times 10^{6} \mathrm{PFU} \mathrm{ml}{ }^{-1}\right)$ or SaPI1 $\left(\sim 3.0 \times 10^{7} \mathrm{PFU} \mathrm{ml}{ }^{-1}\right)$, respectively (Supplementary Table S1), incubated for 15 min at $37^{\circ} \mathrm{C}$, diluted, and plated on BM agar supplemented with appropriate antibiotics.

For $\Phi 187-$-mediated SaPIbov1 transfer, the PS187-H strain producing RboP and GroP WTA was transduced by $\Phi 11$ with SaPIbov1. The resulting strain VW1 was infected with $\Phi 187$ and $100 \mu \mathrm{l}$ of the resulting lysate $\left(\sim 1.3 \times 10^{10} \mathrm{PFU} \mathrm{ml}^{-1}\right)$ was used to infect recipient strains as mentioned above. Note that for $\Phi 187$-mediated SaPIbov1 transduction of Listeria strains, the used lysate contained only $\sim 5.0 \times 10^{5} \mathrm{PFU} \mathrm{ml}^{-1}$. For $\Phi 187$-mediated SaPI187 $\beta$ transfer, SaPI187 $\beta$ was labelled with the ermB resistance marker in S. aureus PS187 wild type resulting in strain VW7, which was subsequently infected with $\Phi 187$ and $100 \mu$ lof the obtained lysate $\left(\sim 3.0 \times 10^{7} \mathrm{PFU} \mathrm{ml}^{-1}\right)$ was used to infect recipient strains as mentioned above. To exclude that transductants resulted from spontaneous uptake of nicked DNA, SaPI-containing lysates were treated with $20 \mathrm{U}$ DNase I for $1 \mathrm{~h}$ at $37^{\circ} \mathrm{C}$ and used for SaPI transfer experiments. For plasmid transduction, pT181-bearing RN4220 was infected with $\Phi 80 \alpha$ and the lysate was used to infect recipient strains. Approximately $8.0 \times 10^{7}$ bacteria were resuspended in phage buffer $(100 \mathrm{mM}$ $\mathrm{MgSO}_{4}, 100 \mathrm{mM} \mathrm{CaCl} 2,1 \mathrm{M}$ Tris- $\mathrm{HCl}, \mathrm{pH} 7.8,0.59 \% \mathrm{NaCl}, 0.1 \%$ gelatine) and mixed with $100 \mu$ l lysate $\left(\sim 1.1 \times 10^{8} \mathrm{PFU} \mathrm{ml}^{-1}\right)$, incubated for $10 \mathrm{~min}$ at $37^{\circ} \mathrm{C}$, mixed with soft agar and poured onto BM agar plates containing $12.5 \mu \mathrm{g} \mathrm{ml}^{-1}$ tetracycline. Transductants were counted after overnight incubation (SaPIs) or after $48 \mathrm{~h}$ at $37^{\circ} \mathrm{C}$ (pT181 and Listeria assays) and transduction efficiency was calculated. SaPI transfer was confirmed by molecular typing of resistance marker.

WTA extraction and purification. WTA was isolated as described previously with minor modifications ${ }^{41}$. Briefly, overnight cultures were washed and disrupted in a cell disrupter (Euler). Cell lysates were incubated at $37^{\circ} \mathrm{C}$ overnight in the presence of DNase and RNase. SDS was added to a final concentration of $2 \%$ followed by ultrasonication for $15 \mathrm{~min}$. Cell walls were washed several times to remove SDS. To release WTA from cell walls samples were treated with $5 \%$ trichloroacetic acid for $4 \mathrm{~h}$ at $65^{\circ} \mathrm{C}$. Peptidoglycan debris was separated via centrifugation $(10 \mathrm{~min}$, $14,000 \mathrm{~g}$ ). Determination of inorganic phosphate as described previously ${ }^{41}$ was used for WTA quantification. These crude WTA extracts were further purified as described previously with minor modifications ${ }^{38}$. Briefly, the $\mathrm{pH}$ of the crude extract was adjusted to 5.5 with $\mathrm{NaOH}$ and dialyzed against water with a Spectra/ Por3 dialysis membrane (MWCO of $3.5 \mathrm{kDa}$; VWR International $\mathrm{GmbH}$, Darmstadt). Samples were concentrated in a SpeedVac concentrator at $45^{\circ} \mathrm{C}$ to $5 \mathrm{ml}$ and applied to DEAE-Sephadex A25 matrix according to Xia et al. ${ }^{38}$ For elution from DEAE-Sephadex A25 matrix $20 \mathrm{mM}$ Tris- $\mathrm{HCl} \mathrm{pH} 7.20 .35 \mathrm{M} \mathrm{NaCl}$ was used. The eluate was dialyzed again against water and concentrated to $1 \mathrm{ml}$. The purified WTA samples were stored at $20^{\circ} \mathrm{C}$ for further analysis. All general analytical chemistry and NMR spectroscopy methods for WTA characterization are described in detail in the supplementary section.

Genome sequencing and analyses. S. aureus PS187 was sequenced initially by Roche 454 pyrosequencing (Roche GS-FLX system), then resequenced by using Illumina technology (Illumina HiSeq2000) at a 150-fold coverage. Details are described in supplementary section.

\section{References}

1. Thomas, C. M. \& Nielsen, K. M. Mechanisms of, and barriers to, horizontal gene transfer between bacteria. Nat. Rev. Microbiol. 3, 711-721 (2005).

2. Frost, L. S., Leplae, R., Summers, A. O. \& Toussaint, A. Mobile genetic elements: the agents of open source evolution. Nat. Rev. Microbiol. 3, 722-732 (2005).

3. Malachowa, N. \& DeLeo, F. R. Mobile genetic elements of Staphylococcus aureus. Cell. Mol. Life Sci. 67, 3057-3071 (2010).

4. Lindsay, J. A. Genomic variation and evolution of Staphylococcus aureus. Int. J. Med. Microbiol. 300, 98-103 (2010).

5. Chambers, H. F. \& Deleo, F. R. Waves of resistance: Staphylococcus aureus in the antibiotic era. Nat. Rev. Microbiol. 7, 629-641 (2009).

6. Mediavilla, J. R., Chen, L., Mathema, B. \& Kreiswirth, B. N. Global epidemiology of community-associated methicillin-resistant Staphylococcus aureus (CA-MRSA). Curr. Opin. Microbiol. 15, 588-595 (2012).

7. Morikawa, K. et al. Expression of a cryptic secondary sigma factor gene unveils natural competence for DNA transformation in Staphylococcus aureus. PLoS Pathog. 8, e1003003 (2012).

8. Xia, G. et al. Wall teichoic acid-dependent adsorption of staphylococcal siphovirus and myovirus. J. Bacteriol. 193, 4006-4009 (2011).

9. Weidenmaier, C. \& Peschel, A. Teichoic acids and related cell-wall glycopolymers in Gram-positive physiology and host interactions. Nat. Rev. Microbiol. 6, 276-287 (2008). 
10. Brown, S. et al. Methicillin resistance in Staphylococcus aureus requires glycosylated wall teichoic acids. Proc. Natl. Acad. Sci. USA 109, 18909-18914 (2012).

11. Endl, J., Seidl, P. H., Fiedler, F. \& Schleifer, K. H. Determination of cell wall teichoic acid structure of staphylococci by rapid chemical and serological screening methods. Arch. Microbiol. 137, 272-280 (1984).

12. Tallent, S. M., Langston, T. B., Moran, R. G. \& Christie, G. E. Transducing particles of Staphylococcus aureus pathogenicity island SaPI1 are comprised of helper phage-encoded proteins. J. Bacteriol. 189, 7520-7524 (2007).

13. Novick, R. P., Christie, G. E. \& Penades, J. R. The phage-related chromosomal islands of Gram-positive bacteria. Nat. Rev. Microbiol. 8, 541-551 (2010).

14. Otto, M. Coagulase-negative staphylococci as reservoirs of genes facilitating MRSA infection: staphylococcal commensal species such as Staphylococcus epidermidis are being recognized as important sources of genes promoting MRSA colonization and virulence. Bioessays 35, 4-11 (2012).

15. Perichon, B. \& Courvalin, P. VanA-type vancomycin-resistant Staphylococcus aureus. Antimicrob. Agents. Chemother. 53, 4580-4587 (2009).

16. Corvaglia, A. R. et al. A type III-like restriction endonuclease functions as a major barrier to horizontal gene transfer in clinical Staphylococcus aureus strains. Proc. Natl. Acad. Sci. USA 107, 11954-11958 (2010).

17. Waldron, D. E. \& Lindsay, J. A. Saul: a novel lineage-specific type I restrictionmodification system that blocks horizontal gene transfer into Staphylococcus aureus and between $S$. aureus isolates of different lineages. J. Bacteriol. 188, 5578-5585 (2006)

18. Monk, I. R., Shah, I. M., Xu, M., Tan, M. W. \& Foster, T. J. Transforming the untransformable: application of direct transformation to manipulate genetically Staphylococcus aureus and Staphylococcus epidermidis. mBio 3 pii e00277-11 (2012).

19. Marraffini, L. A. \& Sontheimer, E. J. CRISPR interference limits horizontal gene transfer in staphylococci by targeting DNA. Science 322, 1843-1845 (2008).

20. Tormo, M. A. et al. Staphylococcus aureus pathogenicity island DNA is packaged in particles composed of phage proteins. J. Bacteriol. 190, 2434-2440 (2008).

21. Asheshov, E. A. \& Jevons, M. P. The effect of heat on the ability of a host strain to support the growth of a Staphylococcus phage. J. Gen. Microbiol. 31, 97-107 (1963).

22. Francois, P. et al. Evaluation of three molecular assays for rapid identification of methicillin-resistant Staphylococcus aureus. J. Clin. Microbiol. 45, 2011-2013 (2007).

23. Holtfreter, S. et al. Clonal distribution of superantigen genes in clinical Staphylococcus aureus isolates. J. Clin. Microbiol. 45, 2669-2680 (2007).

24. Rosenblum, E. D. \& Tyrone, S. Serology, Density, and Morphology of Staphylococcal Phages. J. Bacteriol. 88, 1737-1742 (1964).

25. Meyer, W. A proposal for subdividing the species Staphylococcus aureus. Int. J. Syst. Evol. Microbiol. 17, 387-389 (1967).

26. McCarthy, A. J. et al. The distribution of mobile genetic elements (MGEs) in MRSA CC398 is associated with both host and country. Genome Biol. Evol. 3, 1164-1174 (2011).

27. Mongkolrattanothai, K., Boyle, S., Murphy, T. V. \& Daum, R. S. Novel non$m e c A$-containing staphylococcal chromosomal cassette composite island containing $p b p 4$ and $\operatorname{tagF}$ genes in a commensal staphylococcal species: a possible reservoir for antibiotic resistance islands in Staphylococcus aureus. Antimicrob. Agents. Chemother. 48, 1823-1836 (2004).

28. Gill, S. R. et al. Insights on evolution of virulence and resistance from the complete genome analysis of an early methicillin-resistant Staphylococcus aureus strain and a biofilm-producing methicillin-resistant Staphylococcus epidermidis strain. J. Bacteriol. 187, 2426-2438 (2005).

29. Chen, J. \& Novick, R. P. Phage-mediated intergeneric transfer of toxin genes. Science 323, 139-141 (2009).

30. Bychowska, A. et al. Chemical structure of wall teichoic acid isolated from Enterococcus faecium strain U0317. Carbohydr. Res. 346, 2816-2819 (2011).

31. Geiss-Liebisch, S. et al. Secondary cell wall polymers of Enterococcus faecalis are critical for resistance to complement activation via mannose-binding lectin. J. Biol. Chem. 287, 37769-37777 (2012).

32. Ubeda, C. et al. Antibiotic-induced SOS response promotes horizontal dissemination of pathogenicity island-encoded virulence factors in staphylococci. Mol. Microbiol. 56, 836-844 (2005).
33. Suzuki, T., Swoboda, J. G., Campbell, J., Walker, S. \& Gilmore, M. S. In vitro antimicrobial activity of wall teichoic acid biosynthesis inhibitors against Staphylococcus aureus isolates. Antimicrob. Agents. Chemother. 55, 767-774 (2011).

34. Bae, T. \& Schneewind, O. Allelic replacement in Staphylococcus aureus with inducible counter-selection. Plasmid 55, 58-63 (2006).

35. Bruckner, R. A series of shuttle vectors for Bacillus subtilis and Escherichia coli. Gene 122, 187-192 (1992).

36. Promadej, N., Fiedler, F., Cossart, P., Dramsi, S. \& Kathariou, S. Cell wall teichoic acid glycosylation in Listeria monocytogenes serotype $4 \mathrm{~b}$ requires gtcA, a novel, serogroup-specific gene. J. Bacteriol. 181, 418-425 (1999).

37. Goerke, C. et al. Diversity of prophages in dominant Staphylococcus aureus clonal lineages. J. Bacteriol. 191, 3462-3468 (2009).

38. Xia, G. et al. Glycosylation of wall teichoic acid in Staphylococcus aureus by TarM. J. Biol. Chem. 285, 13405-13415 (2010).

39. Pantucek, R. et al. Identification of bacteriophage types and their carriage in Staphylococcus aureus. Arch. Virol. 149, 1689-1703 (2004).

40. Christie, G. E. et al. The complete genomes of Staphylococcus aureus bacteriophages 80 and 80alpha--implications for the specificity of SaPI mobilization. Virology 407, 381-390 (2010)

41. Weidenmaier, C. et al. Role of teichoic acids in Staphylococcus aureus nasal colonization, a major risk factor in nosocomial infections. Nat. Med. 10, 243-245 (2004).

42. Schleifer, K. H. \&. Fischer, U. Description of a new species of the genus Staphylococcus: Staphylococcus carnosus. Int. J. Syst. Bacteriol. 32, 153-156 (1982).

43. Fiedler, F. Biochemistry of the cell surface of Listeria strains: a locating general view. Infection 16(Suppl 2): S92-S97 (1988).

\section{Acknowledgements}

We thank Jacques Schrenzel, Gabriele Bierbaum, Christiane Wolz, Angelika Gründling Matthias Marschal and Ian Monk for providing bacterial strains and phages and Peter Bauer and Claudia Bauer for help with genome sequencing. This work was supported by grants TRR34 to A.P., B.M.B., and T.D. and SFB766 to G.X. and A.P. from the German Research Council, grants from the German Center for Infectious Disease Research to A.P. and G.X., and SkinStaph and Menage grants to A.P. from the German Ministry of Education and Research.

\section{Author contributions}

V.W., C.L., P. S.C., G.X., M.S. and M.M. performed the experiments and analysed the data; B.M.B. and J.R.P. provided essential materials; V.W., J.R.P., U.N., O.H., T.D., A.P. and G.X. conceived the study; V.W., A.P. and G.X. wrote the manuscript

\section{Additional information}

Accession codes: The Whole Genome Shotgun project has been deposited at DDBJ/ EMBL/GenBank under the accession number ARPA00000000 (BioProject PRJNA197438). The version described in this paper is the first version, ARPA01000000.

Supplementary Information accompanies this paper at http://www.nature.com/ naturecommunications

Competing financial interests: The authors declare no competing financial interests.

Reprints and permission information is available online at http://npg.nature.com/ reprintsandpermissions/

How to cite this article: Winstel, V. et al. Wall teichoic acid structure governs horizontal gene transfer between major bacterial pathogens. Nat. Commun. 4:2345 doi: 10.1038/ ncomms3345 (2013)

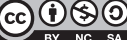

This work is licensed under a Creative Commons AttributionNonCommercial-ShareAlike 3.0 Unported License. To view a copy of this license, visit http://creativecommons.org/licenses/by-nc-sa/3.0/ 